\title{
Dynamic deformation and fracture of single crystal silicon: fracture modes, damage laws, and anisotropy
}

\author{
J. Y. Huang ${ }^{\mathrm{a}, \mathrm{b}, \mathrm{c}}$, J. C. E ${ }^{\mathrm{a}}$, J. W. Huang ${ }^{\mathrm{a}}$, T. Sun ${ }^{\mathrm{d}}$, K. Fezzaa ${ }^{\mathrm{d}}$, S. L. Xu ${ }^{\mathrm{c}, *}$, S. N. Luo ${ }^{\mathrm{a}, \mathrm{b}, *}$ \\ ${ }^{a}$ The Peac Institute of Multiscale Sciences, Chengdu, Sichuan 610031, P. R. China \\ ${ }^{b}$ Key Laboratory of Advanced Technologies of Materials, Ministry of Education, Southwest Jiaotong University, Chengdu, Sichuan 610031, P. R. China \\ ${ }^{c}$ CAS Key Laboratory of Materials Behavior and Design, Department of Modern Mechanics, University of Science and Technology of China, Hefei, Anhui 230027, \\ P. R. China \\ ${ }^{d}$ Advanced Photon Source, Argonne National Laboratory, Argonne, Illinois 60439, USA
}

\begin{abstract}
Impact fracture of single-crystal $\mathrm{Si}$ is critical to long-term reliability of electronic devices and solar cells for its wide use as components or substrates in semiconductor industry. Single-crystal Si is loaded along two different crystallographic directions with a split Hopkinson pressure bar integrated with an in situ x-ray imaging and diffraction system. Bulk stress histories are measured, simultaneously with x-ray phase contrast imaging (XPCI) and Laue diffraction. Damage evolution is quantified with grayscale maps from XPCI. Single-crystal Si exhibits pronounced anisotropy in fracture modes, and thus fracture strengths and damage evolution. For loading along [1 $1 \overline{1} 0]$ and viewing along [001] directions, $(\overline{1} \overline{1} 0)[1 \overline{1} 0]$ cleavage is activated and induce horizontal primary cracks followed by perpendicular wing cracks. However, when loading along [011] and viewing along [111] directions, random nucleation and growth of shear and tensile-splitting crack networks lead to catastrophic failure of materials with no cleavage. The primarywing crack mode leads to a lower characteristic fracture strength due to predamage, but a more concentrated strength distribution, i.e., a higher Weibull modulus, compared to the second loading case. Moreover, the sequential primary cracking, wing cracking and wing-crack coalescence processes result in a gradual increase of damage with time, deviating from theoretical predictions. Particle size and aspect ratios of fragments are discussed with postmortem fragment analysis, which verifies fracture modes observed in XPCI.
\end{abstract}

Keywords: single-crystal Si; dynamic fracture; cleavage; x-ray imaging; Laue diffraction.

\section{Introduction}

Single crystal Si has been widely used as components or substrates in semiconductor industry, such as integrated circuits, solar cells and micro-electromechanical systems [1-5]. Understanding impact-induced deformation, damage and fracture of such materials, including phenomena and underlying mechanisms, is critical for better impact resistance, and long-term reliability of electronic devices. Si is also an prototypical brittle solid of fundamental interest in materials science and mechanics. As regards fracture of single crystal $\mathrm{Si}$, previous studies focused on indentation fracture or phase transition [6, 7], or single-crack propagation in a notched or pre-cleaved sample under quasi-static tension [8-10]. However, dynamic compression fracture, including fracture strengths, fracture modes and their orientation dependence $[9,11]$, have been scarcely discussed.

Extensive investigations have been devoted to compression fracture of such brittle materials as ceramics [12-15], quartz [16], and ice [17, 18]. Under quasi-static loading, the fracture modes can be resolved with the high-speed optical imaging method $[19,20]$ or postmortem SEM analysis [14, 21]. Crack

\footnotetext{
${ }^{*}$ Corresponding author

Email addresses: slxu99@ustc.edu.cn (S. L. Xu ), sluo@pims.ac.cn (S. N. Luo )
}

features such as mirror, mist, hackle and branching, were widely observed in the fracture plane of brittle materials when the stress intensity or crack propagation velocity exceeds a critical value $[10,21]$. Transgranular fracture was typically expected in polycrystalline ceramics $[13,14]$, while intergranular or cleavage fracture, in ice and quartz [18, 22]. However, fracture processes under dynamic loading are highly transient, and it is difficult to resolve crack initiation, growth and coalescence due to motion blur or catastrophic failure [16, 22]. Lamberson et al. [16] conducted experiments on dynamic fracture of transparent quartz crystals with high-speed optical imaging in a reflection mode. Complex interactions of reflected light from microcracks blurred images, and statistical analysis based on the grayscale distribution was used to quantify the damage extent. However, obtaining temporally and spatially resolved damage and fracture dynamics in nontransparent materials has been an experimental challenge [23].

$\mathrm{X}$-ray phase contrast imaging (XPCI) is advantageous for resolving dynamic fracture and fragmentation of nontransparent brittle materials owing to its edge enhancement, strong penetration capability, and high temporal $(\mu \mathrm{s})$ and spatial $(\mu \mathrm{m})$ resolutions [24-27]. The diffraction spots yield 3D information on lattice deformation or rotation $[28,29]$, beneficial to understanding of micro-scale deformation and damage of material- 
s [30-32]. Simultaneous dynamic x-ray imaging and diffraction have been established at the beamline 32-ID of the Advanced Photon Source (APS) [32-34], with a split Hopkinson pressure/tension bar (SHPB/SHTB) implemented for dynamic loading. Dynamic deformation of various materials such as $\mathrm{Mg}$ alloys, metal composites, and granular materials have been investigated with the digital image correlation and Laue diffraction [32, 33, 35-37]. The simultaneous x-ray imaging and diffraction technique is rarely exploited for studying mechanical properties of single crystals during dynamic loading.

In the present work, dynamic fracture strengths and cracking modes of Si single crystals are revealed with multi-scale measurements, i.e., bulk stress histories, mesoscopic XPCI and SEM, and microscopic X-ray Laue diffraction. Dynamic fracture modes of Si along different crystallographic directions are resolved with in situ XPCI, while simultaneous diffraction provides complementary information on lattice-scale deformation and damage. Particle size distributions and aspect ratios of fragments are discussed with postmortem SEM analysis, which verifies fracture modes observed in XPCI. Single-crystal Si exhibits pronounced anisotropy in fracture modes (cleavage or otherwise), and strength distributions (concentrated or scattered). Statistical analysis of XPCI graphs yields evolution of macroscopic damage, deviating from theoretical predictions of the Grady-Kipp model [38] which does not consider microstructures of materials. Our results provide a solid experimental example which can be helpful for developing multiscale, physics-based, damage models of brittle single crystals.

\section{Experimental}

Single-crystal Si in current experiments has an initial density of $2.4 \times 10^{3} \mathrm{~kg} \mathrm{~m}^{-3}$. Samples of types \#1 and \#2 (denoted with subscripts 1 and 2, respectively) are loaded along two different directions, and the corresponding crystallographic orientation$\mathrm{s}$ and loading geometries are shown in Fig. 1. The coordinate system is defined as follows. The $x$-axis is along the bar or loading direction, the $z$-axis is along the $\mathrm{x}$-ray beam direction, and the $y$-axis is perpendicular to the $x$ - and $z$-axes.. The uncertainties in the orientations along the $z$-axis are $\pm 0.5^{\circ}$, while those for the $x$ - and $y$-axes are $\pm 1^{\circ}$. Based on the Hooke's law, the Young's modulus $E_{h k l}$ along the normal of a $(h k l)$ plane can be derived from the elastic compliance matrix $S_{i j}$ as [15]

$$
\frac{1}{E_{h k l}}=S_{11}+\frac{\left(2 S_{12}-2 S_{11}+S_{44}\right)\left(h^{2} l^{2}+k^{2} l^{2}+h^{2} k^{2}\right)}{\left(h^{2}+k^{2}+l^{2}\right)^{2}}
$$

where $S_{11}=0.0077 \mathrm{GPa}^{-1}, S_{12}=-0.0021 \mathrm{GPa}^{-1}$, and $S_{44}=$ $0.0125 \mathrm{GPa}^{-1}$ for $\mathrm{Si}$ [39].

The schematic setup for the split Hopkinson pressure bar (SHPB) loading, and simultaneous, high-speed, x-ray imaging and diffraction system is presented in Fig. 1. The experiments are conducted at the beamline 32-ID-B of the Advanced Photon Source (APS). The probe $\mathrm{x}$-rays are from an APS undulator A light source at a gap of $23 \mathrm{~mm}$, with three main harmonics. The first harmonic is centered at $11.3 \mathrm{keV}$ with a full width at



Figure 1: Schematic experimental setup for split Hopkinson pressure bar (SHPB) loading, and simultaneous, high-speed, x-ray imaging and diffraction. 1: Striker; 2: incident bar; 3: sample; 4: transmission bar; 5: strain gauge; 6: imaging scintillator $\left(\mathrm{Lu}_{3} \mathrm{Al}_{5} \mathrm{O}_{12}: \mathrm{Ce}\right.$ or LuAG:Ce); 7: imaging camera; 8: diffraction scintillator $\left(\mathrm{Lu}_{2-2 x} \mathrm{Y}_{2 x} \mathrm{SiO}_{5}: \mathrm{Ce}\right)$ with illustrative diffraction rings; 9: microchannel plate (MCP); 10: diffraction camera. Inset shows crystallographic orientations of samples of types \#1 and \#2. The $x$-axis is along the bar or loading direction, the $z$-axis is along the $\mathrm{x}$-ray beam direction, and the $y$-axis is perpendicular to the $x$ - and $z$-axes.

half maximum (FWHM) of $0.4 \mathrm{keV}$ [32]. The sample (3) dimensions perpendicular to the incident $\mathrm{x}$-ray beam $\left(O O^{\prime}\right.$ or the $z$-axis) are $3 \mathrm{~mm} \times 3 \mathrm{~mm}$, and the thickness along the view direction is $1 \mathrm{~mm}$. A miniature SHPB device (1, 2, 4 and 5) is implemented along with simultaneous high-speed x-ray imaging $(6,7)$ and diffraction $(8,9,10)$. Details of the imaging and diffraction system were presented previously [32].

The striker (1), incident bar (2) and transmission bar (4) of SHPB are all made of high-strength steel with a diameter of 4 $\mathrm{mm}$. After SHPB's gas gun is fired, impact of the striker on the incident bar generates an elastic wave propagating through the incident bar (along the $x$-axis, Fig. 1). A copper pulse shaper is used to obtain a rising slope of the incident wave [40]. When the incident wave arrives at the interface between the incident bar and the sample (3), it is partially reflected owing to impedance mismatch, while the rest is transmitted into the transmission bar. The incident, reflected and transmitted waves are recorded by strain gages (5). The transmitted and reflected waves are used to calculate the engineering stress $(\sigma)$ and strain rate $(\dot{\varepsilon})$ applied to the sample with the two-wave technique [14, 41], and

$$
\begin{gathered}
\sigma_{\mathrm{s}}=-E_{\mathrm{b}} \varepsilon_{\mathrm{t}} \frac{A_{\mathrm{b}}}{A_{\mathrm{s}}}, \\
\dot{\varepsilon}_{\mathrm{s}}=-\frac{2 C_{\mathrm{b}}\left(\varepsilon_{\mathrm{i}}-\varepsilon_{\mathrm{t}}\right)}{L_{\mathrm{s}}},
\end{gathered}
$$

where $\varepsilon$ is strain, $A$ is cross-section, $L$ is the length, and $C$ is the sound speed. Subscripts $\mathrm{i}$ and $\mathrm{t}$ denote the incident and transmission bar, respectively; subscripts $b$ and $s$ denote bar and sample, respectively. In this work, compressional stress 
and strain are positive.

Eq. (3) is valid when dynamic stress equilibrium is achieved in the sample subjected to SHPB loading, which requires three to five wave reverberations [42]. For $\mathrm{Si}, E_{110}$ is calculated as $170 \mathrm{GPa}$, and the corresponding 1D stress wave velocity is $\sim 8400 \mathrm{~m} / \mathrm{s}$, so the equilibration time is less than $4 \mu \mathrm{s}$. Three-wave analysis [42] also shows that stress equilibrium is achieved after about $5 \mu \mathrm{s}$. The total strain (mainly elastic) integrated with $\dot{\varepsilon}_{\mathrm{s}}$ from time $t=0$ is not appropriate for brittle materials typically failing at very small strains $(<0.5 \%)$ [41]. Therefore, only stress-time curves are presented and discussed where appropriate, as in literature $[16,23]$. The incident wave signal is also used to trigger the high-speed cameras $(7,10)$. Upon loading, the x-rays transmitted through the Si sample form images on a scintillator (6) which are captured by the imaging camera (7) as image sequences. The diffraction signal is captured by another scintillator (8), magnified by a micro channel plate (9) and then recorded by the diffraction camera (10).

\section{Results}

\subsection{Compressional fracture strengths}

Fracture strengths of brittle materials generally exhibit considerable divergence. A Weibull model has been widely used to deal with the scatter in the apparent fracture strength, $\sigma_{\mathrm{f}}$, directly obtained from the compression curves [15, 19, 43, 44]. It assumes that $\sigma_{\mathrm{f}}$ and the probability of failure $\left(P_{\mathrm{f}}\right)$ follow a Weibull distribution,

$$
P_{\mathrm{f}}=1-\exp \left[-\left(\frac{\sigma_{\mathrm{f}}}{\sigma_{0}}\right)^{m}\right],
$$

where $\sigma_{0}$ is the characteristic fracture strength, statistically proportional to the mean fracture strength [43]. $m$ is the Weibull modulus, and describes the extent of scatter in fracture strengths [44].

The characteristic fracture strength and Weibull modulus can be obtained by a linear fitting to the $P_{\mathrm{f}}\left(\sigma_{\mathrm{f}}\right)$ data with

$$
\ln \ln \frac{1}{1-P_{\mathrm{f}}}=m \ln \sigma_{\mathrm{f}}+\ln \frac{1}{\sigma_{0}},
$$

as presented in the inset of Fig. 2. In experiments, $P_{\mathrm{f}}$ is commonly approximated by $\frac{i}{n+1}$, where $n$ is the total number of tests, and $i$ is the ranking of a test based on the strength data sorted in an ascending order. $\sigma_{\mathrm{f}}$ is taken as the extreme stress (not necessarily maximum stress for multiple fracture) before a catastrophic stress drop in samples.

The fracture strengths of single crystal $\mathrm{Si}$ at strain rates of 500-700 s $\mathrm{s}^{-1}$ for sample along different crystal directions are plotted in Fig. 2, along with fitting with the Weibull distribution (Eq. (4)). Sample type \#1 ( $\left.m=8.6, \sigma_{0}=1184 \mathrm{MPa}\right)$ exhibit$\mathrm{s}$ a higher Weibull modulus, but a lower characteristic fracture strength than sample type \#2 $\left(m=5.5, \sigma_{0}=1424 \mathrm{MPa}\right)$. The Weibull moduli are comparable to those of ceramics [15]. The fracture strengths $\left(\sigma_{\mathrm{f}}\right)$ measured here are similar to those for $\mathrm{Si}$ $\langle 110\rangle(0.6-1.2 \mathrm{GPa})$ [45]. However, the discrepancy in fracture strength distributions between sample types \#1 and \#2 is

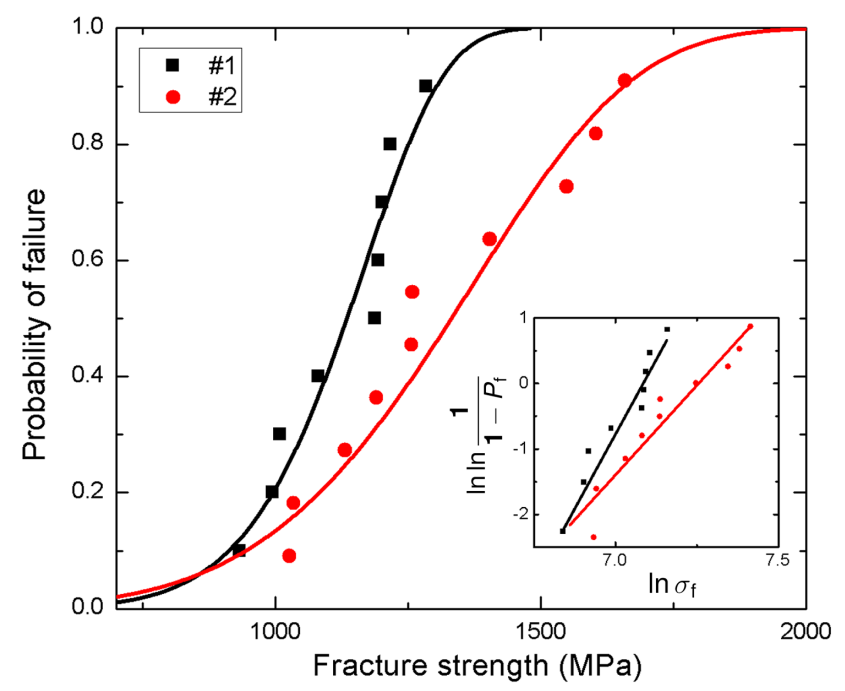

Figure 2: Probability of failure versus compressional fracture strength of single crystal $\mathrm{Si}$ : experimental results (symbols) and Weibull analysis (curves). Sample type \#1: $m=8.6, \sigma_{0}=1184 \mathrm{MPa}$; type \#2: $m=5.5, \sigma_{0}=1424 \mathrm{MPa}$.

actually attributed to anisotropic fracture modes, and will be discussed in detail in Section 4.

\subsection{Simultaneous $x$-ray imaging and diffraction}

Two representative stress-time curves, along with the strainrate histories (after stress equilibrium is achieved), for two loading directions are presented in Figures 3(a) and (b). The strain rate history of sample type $\# 1, \dot{\varepsilon}_{\mathrm{s} 1}(t)$, remains approximately constant at $550 \mathrm{~s}^{-1}$ after $t=6.5 \mu \mathrm{s}$, while $\dot{\varepsilon}_{\mathrm{s} 2}(t)$ shows small fluctuations around a mean of $610 \mathrm{~s}^{-1}$ from $t=6.5$ to $15 \mu \mathrm{s}$. Overall, the two samples exhibit similar responses, i.e. elastic deformation-brittle fracture under compression, but do show differences in details. The compression curve of sample type \#1 shows two distinct inflection points, A and C, at $t=11 \mu \mathrm{s}$ and $22 \mu \mathrm{s}$, respectively. Since the strain rate is approximately constant around point $\mathrm{A}$, the decrease in the slope indicates a modulus reduction and thus softening. After the stress exceeds the fracture strength $\left(\sigma_{\mathrm{B}}\right)$, two fracture stages are seen separated by point $\mathrm{C}$, differentiated by the rate of stress relaxation. This transition can also be resolved in the strain rate histories. For sample type \#2, there is a small inflection point $\mathrm{D}$ at $t=$ $8 \mu \mathrm{s}$, probably due to slight damage in the sample. Beyond the fracture point E, stress decreases sharply to zero with no inflection between. Therefore, type \#2 samples are subject to catastrophic failure.

High-speed image and diffraction sequences are acquired during dynamic compression (Figs. 3(c) and (d)). The frame rates of the imaging and diffraction cameras are both set at 150,000 frames s${ }^{-1}$. The exposure time is $1 \mu \mathrm{s}$ for imaging, and is $0.4-0.6 \mu \mathrm{s}$ for diffraction. Each pixel on the imaging and diffraction cameras corresponds to $\sim 4 \mu \mathrm{m}$ and $60 \mu \mathrm{m}$, respectively. Given the initial crystallographic orientations and

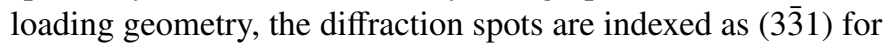

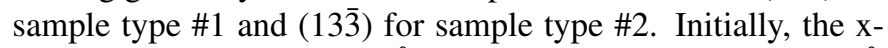
ray wavelengths are $0.572 \AA$ for sample type \#1, and $0.421 \AA$ 

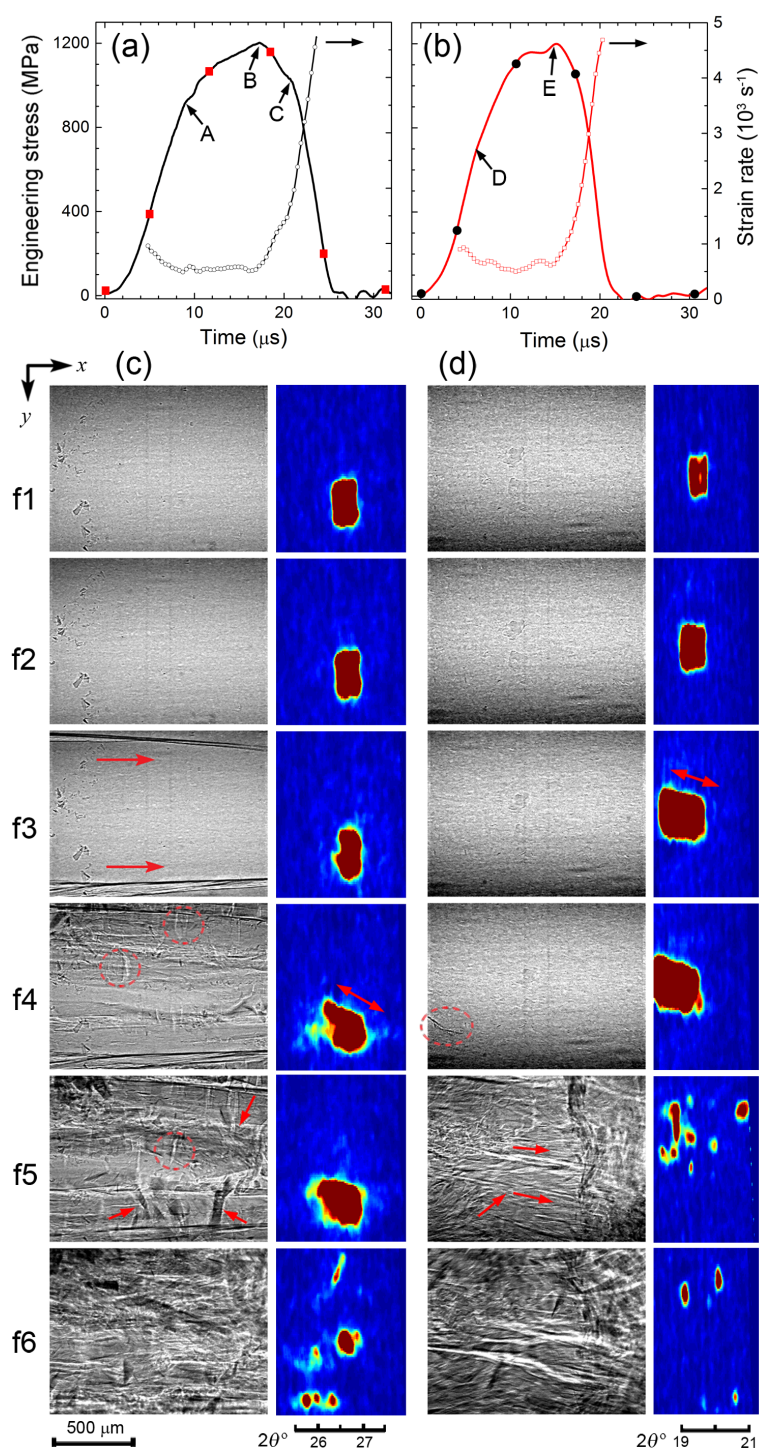

(d)

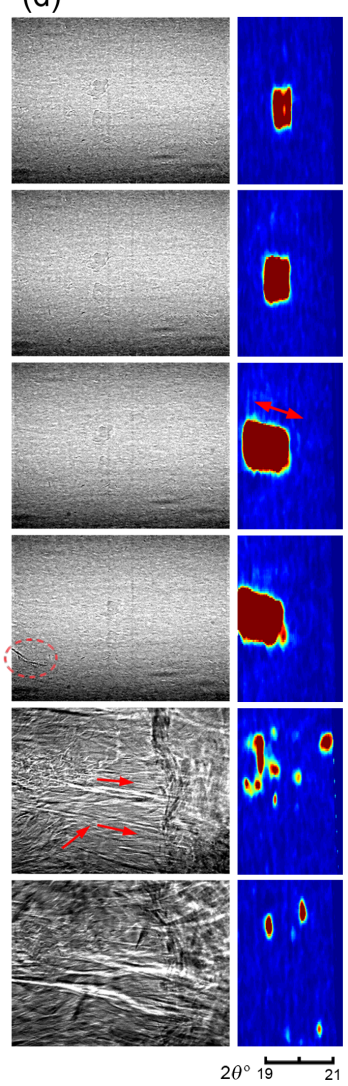

Figure 3: Dynamic deformation and fracture for loading along two crystallographic orientations. (a) and (b) Representative stress and strain-rate histories. (c) and (d) X-ray images (left column) and diffraction patterns (right column) at selected instants marked by rectangles (a) and circles (b) on the corresponding loading curves. (a) and (c) refer to sample type \#1, and (b) and (d), type \#2.

for sample type \#2, located on the second and third harmonics, respectively.

To evaluate lattice strain, the normalized 1D intensity distributions of the diffraction spots are calculated by binning along the $2 \theta$ (Bragg angle) contours [46-48], as shown in Fig. 4 for different instants. The peak shift $\Delta 2 \theta$ can be obtained via Gaussian fitting to the intensity curves [30]. The lattice strain $\varepsilon_{1}$ is calculated from $\Delta 2 \theta$ as

$$
\varepsilon_{1}=1-\frac{\sin \theta_{0}}{\sin \left(\theta_{0}+\Delta 2 \theta\right)},
$$

where $\theta_{0}$ is the diffraction angle for undeformed samples, being $26.582^{\circ}$ and $19.455^{\circ}$ for type \#1 and \#2 samples, respectively.

At current loading conditions, sample type \#1 is subjected to pure elastic compression from frames f1 to f2; consistently, the diffraction spot moves to higher $2 \theta$ while retaining its
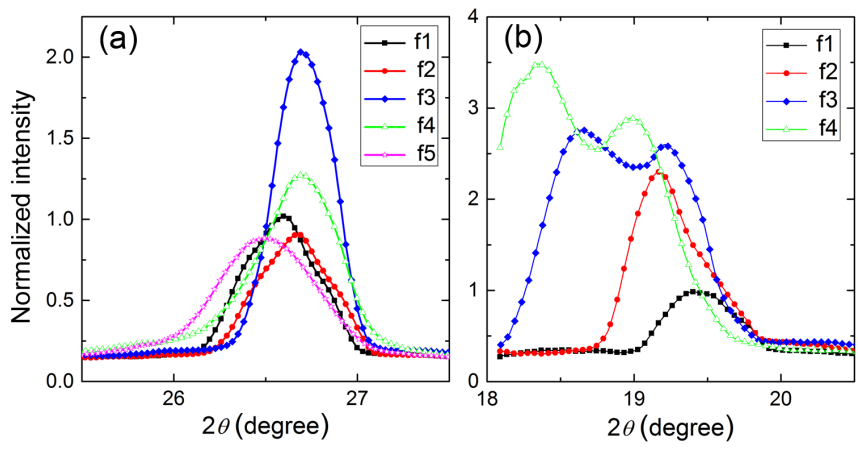

Figure 4: Intensity-normalized 1D diffraction patterns for sample type \#1 (a) and type \#2 (b).

shape [47]. The diffraction spot keeps moving in the same direction at later stages, but its shape and intensity changes, likely due to cracking-induced crystal rotation. The peak shift between frames $\mathrm{f} 1$ and $\mathrm{f} 2$ is $0.075^{\circ}$, and the corresponding lattice strain is $\varepsilon_{112}=0.27 \%$, comparable to the elastic compaction of (3)31) plane $0.23 \%\left(E_{\{331\}}=174 \mathrm{GPa}\right)$. At frame $\mathrm{f} 3$, the imaging shows horizontal primary cracks, which lead to softening as manifested in the bulk-scale compression curve (Fig. 3(a)). The peak shift between frame $\mathrm{f} 1$ and $\mathrm{f} 3$ is $0.128^{\circ}$. The nominal lattice strain between frame $\mathrm{f} 3$ and $\mathrm{f} 1$ is $\varepsilon_{123}=0.47 \%$, lower than the nominal elastic compression strain of $0.59 \%$. A probable reason is that cracking leads to stress release and local crystal rotation, and thus deviation from the apparent elastic deformation as marked by the inflection point A (Fig. 3(a)). At frame f4, additional horizontal cracks form, and micro wing cracks (marked by dashed ellipses) develop perpendicular to the primary cracks, giving rise to the stress drop in the compression curve. Wing cracks supply additional means of energy dissipation $[8,49]$. These wing cracks then coalesce into macrocracks or damage bands (marked by arrows) at frame f5, leading to final failure. Therefore, the second inflection point B may refer to the onset of rapid coalescence of wing cracks and splitting cracks. The corresponding diffraction spots exhibit considerable distortion and peak broadening at frames f3-f5: FWHM in the intensity vs. $2 \theta$ curves (Fig. 4 (a)) increases from $0.368^{\circ}$ at $\mathrm{f} 3$ to $0.522^{\circ}$ at $\mathrm{f} 4$, and $0.623^{\circ}$ at frame f5. Heterogeneous strain distributions across the sample (local stress and strain relaxations due to cracking), and minor lattice/grain (cracks act as "grain boundaries") rotation along the $y$-axis can both contribute to peak broadening in the $2 \theta$ direction [30, 31]. The sample remains as a whole until frame $\mathrm{f} 6$ where the diffraction spot split into several spots, due to the fragments of different orientations formed upon failure.

For sample type \#2, imaging shows no visible damage before frame f3. However, the diffraction spot exhibits pronounced change in shape and peak position. The peak shift from frame f1 to $\mathrm{f} 2$ is $-0.024^{\circ}$. Since the FWHM (Fig. 4(b)) remains approximately constant, rigid body rotation along the $y$-axis due to compression accommodation may contribute to the peak shift to lower $2 \theta$, along with an increase in the spectral flux of incident x-rays. Pronounced peak broadening and splitting are observed at frame f3. The FWHM increases from $0.562^{\circ}$ at $\mathrm{f} 2$ to 
$1.082^{\circ}$ at $\mathrm{f} 3$, and is attributed to heterogeneous strain distributions and/or local lattice rotation induced by microcrack nucleation [31], consistent with the small inflection (point D in the compression curve, Fig. 3(b)). At frame f4, some microcracks develop into a major crack, as marked by the dashed ellipse in Fig. 3(d). The corresponding diffraction spot exhibits peak shift further to lower $2 \theta$, due to cracking-induced crystal rotation. At frame f5, microcracks across the sample evolve into cross-linked splitting and shear cracks, resulting in crack networks and fragmentation. Material fragmentation in turn gives rise to that of the diffraction spot. Sample type \#2 indeed exhibits a microdamamge accumulation followed by catastrophic failure, distinct from sample type \#1, in accord with their compression curves.

\subsection{Damage laws}

Damage evolution of materials is critical to continuum-scale modeling of damage and fracture [16]. We use grayscale values in x-ray images to quantify the extent of damage, after a flatfield correction is applied [50]. The transmitted intensity field $I(x, y, t)$ is corrected to $I_{\mathrm{c}}(x, y, t)$ via

$$
I_{\mathrm{c}}(x, y, t)=\frac{I(x, y, t)}{I(x, y, 0)} I_{0}^{*},
$$

where $I_{0}^{*}$ is the area mean of $I(x, y, 0)$. In the transmission mode, the mean intensity of the $\mathrm{x}$-ray images remains approximately constant. But the spatial distribution of grayscale values, $g(x, y, t)$, becomes increasingly heterogeneous as time evolves. $g(x, y, t)$ is calculated as

$$
g(x, y, t)=I_{\mathrm{c}}(x, y, t)-I_{\mathrm{c}}(t)^{*},
$$

where $I_{\mathrm{c}}(t)^{*}$ is the area mean of $I_{\mathrm{c}}(x, y, t)$.

$g(x, y, t)$ of sample type \#1 is presented in Fig. 5(a) as an example. It is homogeneous at frames $\mathrm{f} 1$ and $\mathrm{f} 2$. Two bright bands appear on the homogeneous background at frame f3, corresponding to central zones of two primary cracks (less dense), while the crack edges form two dark bands due to transient compression. The heterogeneity increases considerably as new wing cracks and splitting cracks develop, and old cracks expand at frames $\mathrm{f} 4-\mathrm{f6}$. We use the normalized standard deviation of grayscale values $(\Phi)$, to quantify the damage extent $(D)$ of the sample, with

$$
\Phi(t)=\sqrt{\frac{1}{a b} \iint g(x, y, t)^{2} \mathrm{~d} x \mathrm{~d} y},
$$

where $a$ and $b$ are the two dimensions of $g(x, y, t)$. It follows that

$$
D(t)=\frac{\Phi(t)-\Phi(0)}{\Phi\left(t_{\mathrm{f}}\right)-\Phi(0)} .
$$

Here $t_{\mathrm{f}}$ refers to the moment of sample failure, defined as the moment when the axial stress in the sample falls below half of the fracture strength, and $D\left(t_{\mathrm{f}}\right)=1$.

Grady and Kipp [38] derived a phenomenological damage model based on the Weibull distribution for dynamic fracture
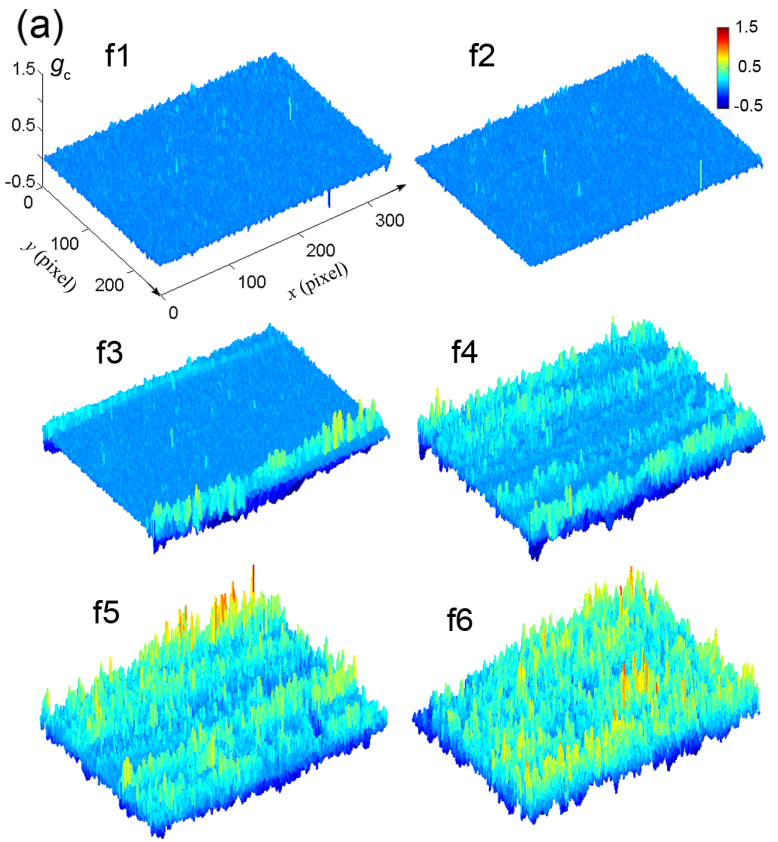

(b)

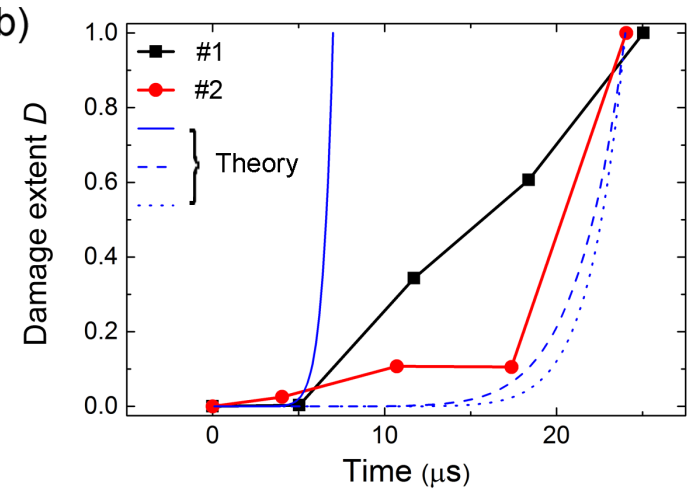

Figure 5: Time evolution of damage in Si single crystals. (a) Grayscale value distributions for sample type \#1. (b) Damage parameter $D$ evolution. Three theoretical curves from the modified Grady-Kipp damage model correspond to: $m=8.6, t_{\mathrm{f}}=7 \mu \mathrm{s}$ (solid line); $m=5.5, t_{\mathrm{f}}=24 \mu \mathrm{s}$ (dashed line); and $m=8.6$, $t_{\mathrm{f}}=24 \mu \mathrm{s}$ (dotted line).

of oil shale. The damage evolution $D(t)$ and final fragment size $(s)$ distribution, $f(s)$, can be described as

$$
\begin{gathered}
D(t)=\frac{8 \pi C_{\mathrm{g}}^{3} k}{(m+1)(m+2)(m+3)} \dot{\varepsilon}^{m} t^{m+3}, \\
f(s)=\frac{\pi m k s^{3}}{12 C_{\mathrm{g}}}\left(t_{\mathrm{f}}-s / 2 C_{\mathrm{g}}\right)^{m-1} \dot{\varepsilon}^{m} .
\end{gathered}
$$

$\dot{\varepsilon}$ is the loading strain rate, and $m$ is the Weibull modulus; $k$ and $C_{\mathrm{g}}$ are fracture constants related to damage nucleation and growth, and $t_{\mathrm{f}}$ depends on these two parameters. Since $D\left(t_{\mathrm{f}}\right)=$ 1, Eq. (11) can be rewritten as

$$
D(t)=\left(\frac{t}{t_{\mathrm{f}}}\right)^{m+3},
$$

and $t_{\mathrm{f}}$ can be directly obtained from the stress-time curves.

The damage evolutions of the two samples obtained from high-speed images are presented in Fig. 5(b), along with three 
curves based on the modified Grady-Kipp damage model (Eq. (13)). The experimental results show that damage is essentially zero before $t=5 \mu \mathrm{s}$. Then, $D$ of sample type \#1 increases approximately linearly from 0 to 1 as time evolves, corresponding to sequential cleavage fracture, wing (or secondary) crack nucleation and coalescence. However, the damage extent of sample type \#2 exhibits a slight increase from 0 to 0.1 at $t=$ $11 \mu \mathrm{s}$ and remains approximately constant until $t=18 \mu \mathrm{s}$. The damage metric is consistent with the diffraction measurements where the diffraction spots show pronounced peak broadening. After that, a sharp increase in damage corresponds to a catastrophic failure (frame f5 in Fig. 3(d)). Si single crystals exhibit pronounced anisotropy in damage evolution when loaded along different crystallographic directions. The Grady-Kipp model captures the catastrophic growth of damage before sample failure in sample type \#2, but not the gradual damage in sample type \#1 and pre-damage in sample type \#2. A probable reason is that such "mean field" models, including the Grady-Kipp model and Paliwal-Ramesh model [51], do not consider anisotropy in damage nucleation and growth modes (e.g. cleavage) in single crystals, and microstructures (e.g. grain boundaries, defects) in polycrystalline solids.

\subsection{Fracture and fragmentation}

Fragments are collected after each dynamic test. Two representative SEM micrographs of the fragments are shown in Figs. 6(a) (sample type \#1) and (b) (sample type \#2), and the corresponding fragment or grain size distributions, in Fig. 6(c). Fragment size is the average of the maximum and minimum lengths of a fragment.

Sample type \#2 has a slightly lager mean grain size than sample type \#1 (97 versus $117 \mu \mathrm{m}$ ), but it has more fine particles $(<50 \mu \mathrm{m})$ and thus exhibits a wider distribution of fragmen$\mathrm{t}$ sizes. This is consistent with the theoretical prediction from Eq. (12) [38] that a lower Weibull modulus of sample type \#2 is expected to yield a broader fragment size distribution. The imaging with a larger observation window of sample type \#2 (in another test), presented in the inset of Fig. 6(c), provides a probable explanation for fines production. A so-called failure wave forms due to coalescence of crack networks [52, 53], under continual loading after cracking and fracture of materials. However, the failure wave cannot propagate far away from the loading end due to fast stress release in the sample after fracture, which explains why failure wave is not observed in a small observation window (Fig. 3). This secondary fragmentation of primary fragments produces more fine particles in sample type \#2 than in sample type \#1, since the failure wave is not observed in sample type \#1.

The Rosin-Rammer distribution (similar to the Weibull distribution) has been widely adopted to describe the cumulative grain size distributions of brittle fragmentation [54], and states that

$$
f(s)=1-\exp \left[-\left(\frac{s}{s_{\mathrm{c}}}\right)^{n}\right],
$$

where $s$ is fragment size, $s_{\mathrm{c}}$ is a characteristic fragment size, and $n$ is a shape factor describing the scattering degree of parti-
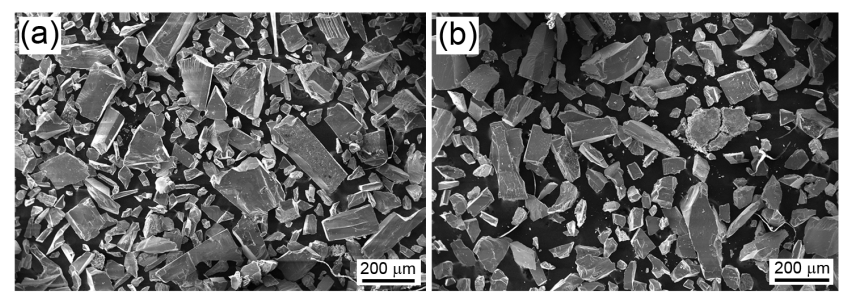

(c)



(d)

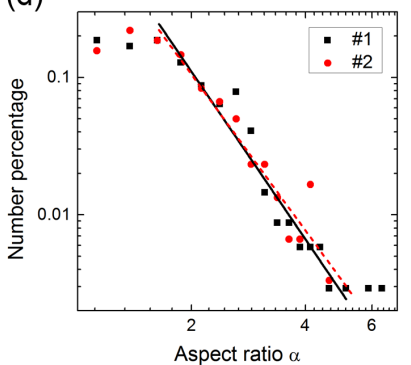

Figure 6: Postmortem SEM micrographs of fragments from sample type \#1 (a) and sample type \#2 (b), and the corresponding grain size distributions (c) and aspect ratio distributions (d). Inset in (c) is a dynamic x-ray image of sample type \#2.

cle sizes. Fitting to the experimental points with Eq. (14) yields $s_{\mathrm{c}}=110 \mu \mathrm{m}$ and $n=3.25$ for sample type \#1, and $s_{\mathrm{c}}=142$ $\mu \mathrm{m}$ and $n=2.4$ for sample type \#2. The phenomenological Rosin-Rammer distribution describes well the experimental results (Fig. 6(c)). At fine particles range, it evolves to a power law with $n$ being the exponent. The shape factor is higher than those previously reported for glass and ceramics (typically $n=0.5-1.5$ ) [54]. This may be attributed to the fracture modes of single crystals different from those of amorphous glasses or polycrystalline ceramics.

Since fragment shapes are closely related to the fracture mechanisms [55], the aspect or slenderness ratio $\alpha$ is used to characterize fragment shapes. For a fragment, $\alpha$ is the ratio of its maximum length to minimum length. The cumulative number distribution of $\alpha$ for sample types \#1 and \#2 are presented in Fig. 6(d). The probability distribution of aspect ratios can be described with a power law: $P(\alpha) \sim\left(\alpha / \alpha_{\mathrm{c}}\right)^{\beta}$, where $\alpha_{\mathrm{c}}$ is a normalization aspect ratio and $\beta$ is used to describe the scatter of $\alpha$. $\beta$ is -4.05 for sample type \#1, and -3.77 for sample type $\# 2$, similar to the values observed in impact fragmentation of hollow glass spheres [55]. Different fracture mechanisms lead to different fragment shapes. The larger $\beta$-value for sample type \#2 indicates that its fracture favors the formation of anisotropic fragments.

The dependence of aspect ratios on fragment size is explored in Fig. 7(a). Fragment size is normalized by the mean grain size. The experimental data of sample type \#1 are divided largely into two groups, corresponding to two different fracture modes (Fig. 7(b)). At small sizes, $\alpha$ exhibits a sharp increase as fragment size increases, while the $\alpha$-values for fragments lager than 1.5 times the mean grain size cluster around 2. However, the experimental data of sample type \#2 are more scattered, possibly due to multiple fracture mechanisms (Fig. 7(c)), and secondary fragmentation caused by the failure wave. The comb- 
(a)

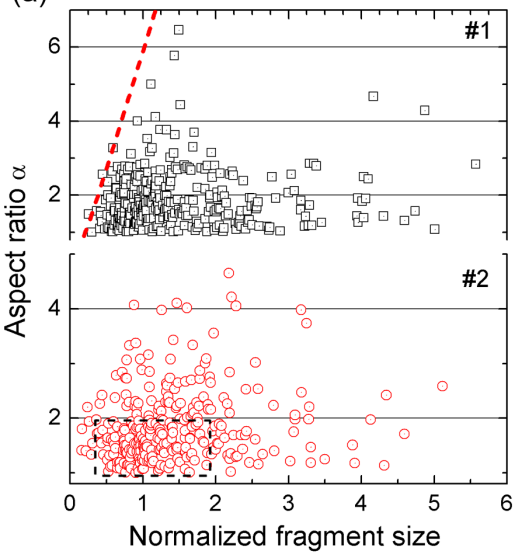

Figure 7: Aspect ratio versus fragment size plots for type \#1 and \#2 samples (a), and corresponding fracture modes (b) and (c).

like crack branching mechanism, as illustrated in Fig. 7(b), was discussed by Renshaw and Schulson [56]. Wing or secondary cracks develop perpendicular to primary cracks, since sliding along the primary cracks generates a stress concentration at the end of the sliding segment. The length $(h)$ and spacing $(w)$ of wing cracks are related to the the length of primary cracks $(2 c)$ and lateral confinement ratio, $R=\sigma_{2} / \sigma_{1}$, as

$$
\begin{gathered}
\frac{w}{c}=\left[1+\left(1-\mu \frac{1+R}{1-R}\right)^{\frac{2}{3}}\right]^{\frac{1}{2}}-1, \\
\frac{h}{c}=\frac{1-R-\mu(1+R)}{4.3 R} .
\end{gathered}
$$

Here $\mu$ is the friction coefficient.

For 1D-stress compression, $R$ equals 0 at the bulk scale. However, $R$ is not necessarily zero locally, e.g. at $100 \mu \mathrm{m} \mathrm{s}-$ cales, especially during dynamic loading $[57,58]$. When the local longitudinal strain acceleration is much higher than the lateral strain acceleration, lateral confinement is incurred [57]. In the extreme case, $1 \mathrm{D}$ stress loading transits to $1 \mathrm{D}$ strain loading, with $R_{\max }=v /(1-v)$. For Si, the Poisson's ratio $v \approx 0.27$ [59], and $R_{\max }=0.37$. In the following, $R$ is used as an intermediate variable to construct a theoretical relationship between the aspect ratio and fragment size. Here, we consider fragments from breakage of microcolumns between adjacent wing cracks. The aspect ratio of fragments is calculated as $\alpha=\frac{h}{w}$, and the normalized fragment size is $\bar{s}=\frac{1}{2}\left(\frac{h}{c}+\frac{w}{c}\right)$. The $\alpha-\bar{s}$ relationship, corresponding to a $R$ from 0.3 to 0.075 , is presented in Fig. 7(a) assuming $\mu=0.4$ (dashed line) [12]. The theoretical line sets physically an upper limit to the aspect ratio of fragments, and exhibits a similar trend to the experimental data at small $\bar{s}$. Therefore, small fragments with high aspect ratios may be attributed to breakage of microcolumns between wing cracks. The wing cracks vary in length and spacing due to heterogeneous strain distributions, and thus produce fragments with various sizes and aspect ratios. However, since multiple primary cracks develop in the material, interval between primary cracks also affects fragment sizes and aspect ratios. When wing cracks emitted from one primary crack encounter another parallel primary crack, large fragments with low or high aspect ratios are produced, depending on the distance of primary cracks and dominant wing cracks.

As sketched in Fig. 7(c) (cf. Fig. 3(d)), the dominant fracture mode of sample type \#2 is the formation of shear cracking networks. The central area of cracking networks (marked by dashed parallelogram) produces fragments with isotropic shapes, mainly of small size. This is consistent with Fig. 7(a) that lots of experimental points locate in the dashed rectangle with aspect ratios lower than 2. However, the primary-wing crack mode also appears in sample type \#2 (see the top right corner of frame f5 in Fig. 3(d)), which produces high aspectratio fragments with small sizes.

\section{Discussions}

Single crystal Si exhibits quite different fracture modes when loaded along different crystallographic directions, which are repeatable in different shots. Such an anisotropy can be attributed largely to cleavage-induced fracture. Previous studies reported cleavage planes $\{111\}$ and $\{110\}$ in single crystal Si [39], and the $\langle 110\rangle$ directions are the easy propagation directions for both types of cleavage planes [60]. Therefore, ( $\overline{1} \overline{1} 0)[1 \overline{1} 0]$ and (111)[110] are probable cleavage systems considering the loading geometry of sample type \#1. Tensile splitting along the $y$ axis induced by the Poisson's effect leads to this cleavage fracture. Since $\{110\}$ has a slightly lower fracture toughness than $\{111\}$ [61], and (111) is not strictly perpendicular to the Poisson expansion direction (Fig. 1 inset), (1̄10)[110] cleavage is more likely to be activated, giving rise to the primary cracks before fracture in sample type \#1. Cleavage fracture is also verified in SEM micrographs of fragments (Fig. 8(a)), showing parallel, uniformly-spaced, step-like crack surfaces. For sample type \#2, (111)[01 $\overline{1}]$ and (111)[ $\overline{2} 11]$ are probable cleavage systems [39], and the cleavage surfaces are on the $x y$-plane. Since the sample area on the $x y$-plane is two times that on the $y z$-plane when cleavage occurs along the [1ㅣㅣㄹ direction (the $y$-axis), the total fracture surface energy for cleavage on the former plane is twice that on the latter plane. That may explain why cleavage fracture is not observed before shear cracking in sample type \#2.

For sample type \#1, cleavage fracture outruns random microdamage, leading to a few primary cracks; the numbers of primary cracks and their propagation directions are both limited, so its Weibull modulus is high. The propagation of a primary cleavage is subjected to little resistance, so its characteristic fracture strength is lower than sample type \#2 (Fig. 2). For sample type \#2, microcracks nucleate "homogeneously", and coalesce and grow into crack networks. Such random microcracks tangle and serve as barriers to the propagation of each other, as opposed to cleavage cracks, giving rise to lower Weibull modulus but higher characteristic fracture strength [62]. In addition, the random damage nucleation and multiple crack modes lead to a wider spread of fragment sizes and thus a wider fragment size distribution (Fig. 6(c)). 

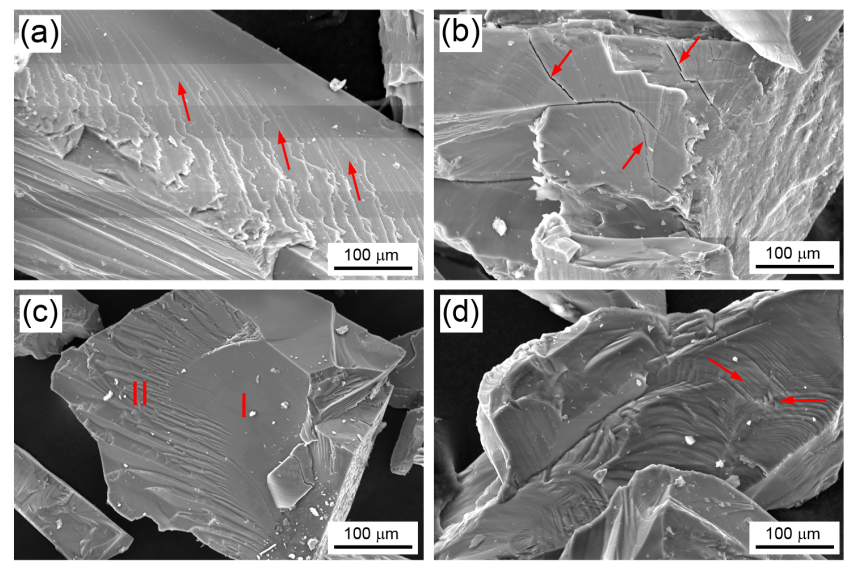

Figure 8: SEM micrographs of recovered fragments.

Grady [63] proposed an analytical model, based on the balance between kinetic energy $T\left(\propto s^{2} ; s\right.$ is the fragment size) and surface energy of newly created fragments $\Gamma\left(\propto s^{-1}\right)$, to predict the mean fragment size $s_{\mathrm{m}}$ as

$$
s_{\mathrm{m}}=\left(\frac{\sqrt{20} K_{\mathrm{IC}}}{\rho C \dot{\varepsilon}}\right)^{\frac{2}{3}},
$$

where $K_{\mathrm{IC}}$ is the fracture toughness, $\rho$ is the density, $C$ is the sonic velocity, and $\dot{\varepsilon}$ is the loading strain rate. For $\mathrm{Si}, K_{\mathrm{IC}}=$ $0.89 \mathrm{MPam}^{\frac{1}{2}}, \rho=2.4 \times 10^{3} \mathrm{kgm}^{-3}, C=8400 \mathrm{~ms}^{-1}$, and $\dot{\varepsilon}=$ $600 \mathrm{~s}^{-1}$. The predicted mean grain size, $4.7 \mathrm{~mm}$, is an order of magnitude higher than that obtained in our experiments.

Shih et al. [15] also observed this discrepancy, and discussed the possible reasons via evaluating actual energy dissipation sources during fragmentation in a brittle solid. They pointed out that apart from kinetic and surface energies, microplasticity around crack tips and internal cracking also play important roles in energy dissipation. It is also true for single-crystal Si. Internal microcracks are widely observed in fragments (indicated by arrows in Fig. 8(b)) besides external surfaces. In addition, fracture surface roughness should be considered; the grooves and wrinkles (Figs. 8(c) and (d)) lead to nonnegligible increase in the effective surface areas. As shown in Fig. 8(c), the fracture surface exhibits two distinct areas: smooth (I) and rough (II), similar to the mirror-hackle transition [8, 10] when the crack velocity exceeds some critical value during propagation. These features are typical for dynamic fracture due to crack instability [64]. Under impact loading, frictional dissipation between sliding segments and fragments should also be considered. Frictional melting has been observed during dynamic fracture of ceramics, silica glass and quartz [65, 66]. Previous experiments have shown that local temperature of $3200 \mathrm{~K}$ for glass, and $4700 \mathrm{~K}$ for quartz may be achieved during crack tip extension [66]. Quantification of the modified energy conservation equation may give a more accurate estimate of the mean particle size. In fact, conservation equations (including mass, momentum and energy) and damage laws compose of a general theoretical damage constitutive model which can predict strength and deformation evolution of materials subject to dy- namic loading.

\section{Conclusions}

Single-crystal $\mathrm{Si}$ is subjected to SHPB loading along two different crystallographic directions, and exhibits pronounced anisotropy in fracture modes, fracture strengths and damage evolution, which is associated with cleavage fracture. For loading along [1 $1 \overline{1} 0]$ and viewing along [001] directions, the ( $\overline{1} 10)[1 \overline{1} 0]$ cleavage system is activated, inducing horizontal primary cracks followed by perpendicular wing cracks. However, random nucleation and growth of shear and tensile-splitting crack networks lead to catastrophic failure of materials with no cleavage for loading along [01 $\overline{1}]$ and viewing along [111] directions. The primary-wing crack mode for the former loading geometry gives rise to a lower characteristic fracture strength due to predamage but a more concentrated strength distribution (a higher Weibull modulus), compared to the second loading geometry. The sequential primary cracking, wing cracking and wing-crack coalescence processes result in a gradual increase of damage with time for the first loading geometry, as opposed to a much more catastrophic failure due to random damage nucleation and growth around initial flaws for the second geometry. Particle size and aspect ratios of fragments analysis verifies fracture modes observed in XPCI. Size dependence of aspect ratios of fragments indicates that the primary-wing crack mode produces small fragments with high aspect ratios, while shear crack networks produce isotropic fragments.

\section{Acknowledgments}

We thank B.X. Bie, D. Fan and L. Lu for the help in X-ray imaging and diffraction experiments. This work is supported by the 973 Project of China (No. 2014CB845904) and NSFC (No. 11472253) of China. Use of the Advanced Photon Source was supported by the U. S. Department of Energy, Office of Science, Office of Basic Energy Sciences, under Contract No. DE-AC02-06CH11357.

\section{References}

[1] D. Y. Khang, H. Jiang, Y. Huang, J. A. Rogers, A stretchable form of single-crystal silicon for high-performance electronics on rubber substrates, Science 311 (2006) 208-212.

[2] A. Masolin, P.-O. Bouchard, R. Martini, M. Bernacki, Thermomechanical and fracture properties in single-crystal silicon, J. Mater. Sci. 48 (2013) 979-988.

[3] W. Kang, M. T. A. Saif, In situ study of size and temperature dependent brittle-to-ductile transition in single crystal silicon, Adv. Funct. Mater. 23 (2013) 713-719.

[4] D. V. Kubair, D. J. Cole, L. C. Ciacchi, S. M. Spearing, Multiscale mechanics modeling of direct silicon wafer bonding, Scripta Mater. 60 (2009) 1125-1128.

[5] A. S. Budiman, G. Illya, V. Handara, W. A. Caldwell, C. Bonelli, M. Kunz, N. Tamura, D. Verstraeten, Enabling thin silicon technologies for next generation c-si solar pv renewable energy systems using synchrotron $\mathrm{x}$ ray microdiffraction as stress and crack mechanism probe, Sol. Energy Mater. Sol. Cells 130 (2014) 303-308. 
[6] Y. B. Gerbig, S. J. Stranick, R. F. Cook, Measurement of residual stress field anisotropy at indentations in silicon, Scripta Mater. 63 (2010) 512515.

[7] H. Huang, J. Yan, New insights into phase transformations in single crystal silicon by controlled cyclic nanoindentation, Scripta Mater. 102 (2015) 35-38.

[8] T. Cramer, A. Wanner, P. Gumbsch, Energy dissipation and path instabilities in dynamic fracture of silicon single crystals, Phys. Rev. Lett. 85 (2000) 788-791.

[9] J. R. Kermode, T. Albaret, D. Sherman, N. Bernstein, P. Gumbsch, M. C Payne, G. Csányi, A. De Vita, Low-speed fracture instabilities in a brittle crystal, Nature 455 (2008) 1224-1227.

[10] M. J. Buehler, H. Tang, A. C. T. van Duin, W. A. Goddard III, Threshold crack speed controls dynamical fracture of silicon single crystals, Phys. Rev. Lett. 99 (2007) 165502

[11] A. Rack, M. Scheel, A. N. Danilewsky, Real-time direct and diffraction X-ray imaging of irregular silicon wafer breakage, IUCrJ 3 (2016) 108114.

[12] H. Wang, K. T. Ramesh, Dynamic strength and fragmentation of hotpressed silicon carbide under uniaxial compression, Acta Mater. 52 (2004) 355-367.

[13] S. Acharya, S. Bysakh, V. Parameswaran, A. K. Mukhopadhyay, Deformation and failure of alumina under high strain rate compressive loading, Ceram. Int. 41 (2015) 6793-6801.

[14] L. Farbaniec, J. D. Hogan, K. T. Ramesh, Micromechanisms associated with the dynamic compressive failure of hot-pressed boron carbide, Scripta Mater. 106 (2015) 52-56.

[15] C. J. Shih, M. A. Meyers, V. F. Nesterenko, S. J. Chen, Damage evolution in dynamic deformation of silicon carbide, Acta Mater. 48 (2000) 2399 2420 .

[16] L. Lamberson, K. T. Ramesh, Spatial and temporal evolution of dynamic damage in single crystal $\alpha$-quartz, Mech. Mater. 87 (2015) 61-79.

[17] N. Golding, E. M. Schulson, C. E. Renshaw, Shear faulting and localized heating in ice: the influence of confinement, Acta Mater. 58 (2010) 5043 5056.

[18] M. Montagnat, P. Duval, P. Bastie, B. Hamelin, Strain gradients and geometrically necessary dislocations in deformed ice single crystals, Scripta Mater. 49 (2003) 411-415.

[19] J. Huang, S. Xu, H. Yi, S. Hu, Size effect on the compression breakage strengths of glass particles, Powder Technol. 268 (2014) 86-94.

[20] S. Xu, J. Huang, P. Wang, C. Zhang, L. Zhou, S. Hu, Investigation of rock material under combined compression and shear dynamic loading: an experimental technique, Int. J. Impact Eng. 86 (2015) 206-222.

[21] Y. L. Tsai, J. J. Mecholsky, Fractal fracture of single crystal silicon, J. Mater. Res. 6 (1991) 1248-1263.

[22] J. Kimberley, K. T. Ramesh, O. S. Barnouin, Visualization of the failure of quartz under quasi-static and dynamic compression, J. Geophys. Res. 115 (2010) B08207.

[23] B. Paliwal, K. T. Ramesh, Effect of crack growth dynamics on the ratesensitive behavior of hot-pressed boron carbide, Scripta Mater. 57 (2007) 481-484.

[24] S. N. Luo, B. J. Jensen, D. E. Hooks, K. Fezzaa, K. J. Ramos, J. D. Yeager, K. Kwiatkowski, T. Shimada, Gas gun shock experiments with single-pulse $\mathrm{x}$-ray phase contrast imaging and diffraction at the Advanced Photon Source, Rev. Sci. Instrum. 83 (2012) 073903

[25] M. Hudspeth, B. Claus, S. Dubelman, J. Black, A. Mondal, N. Parab, C. Funnell, F. Hai, M. Qi, K. Fezzaa, S. N. Luo, W. Chen, High speed synchrotron $\mathrm{X}$-ray phase contrast imaging of dynamic material response to split hopkinson bar loading, Rev. Sci. Instrum. 84 (2013) 025102.

[26] S. Terzi, L. Salvo, M. Suéry, N. Limodin, J. Adrien, E. Maire, Y. Pannier, M. Bornert, D. Bernard, M. Felberbaum, M. Rappaz, E. Boller, In situ Xray tomography observation of inhomogeneous deformation in semi-solid aluminium alloys, Scripta Mater. 61 (2009) 449-452.

[27] J. R. Royer, E. I. Corwin, A. Flior, M.-L. Cordero, M. L. Rivers, P. J. Eng, H. M. Jaeger, Formation of granular jets observed by high-speed X-ray radiography, Nature Phys. 1 (2005) 164-167.

[28] T. Ungár, Microstructural parameters from X-ray diffraction peak broadening, Scripta Mater. 51 (2004) 777-781.

[29] J. W. Huang, J. C. E, J. Y. Huang, T. Sun, K. Fezzaa, S. N. Luo, Dynamic crystal rotation resolved by high-speed synchrotron $\mathrm{X}$-ray laue diffraction, J. Synchrotron Rad. 23 (2016) 712-717.
[30] S. J. Turneaure, Y. M. Gupta, K. Zimmerman, K. Perkins, C. S. Yoo, G. Shen, Real-time microstructure of shocked LiF crystals: use of synchrotron X-rays, J. Appl. Phys. 105 (2009) 053520.

[31] J. M. Winey, J. N. Johnson, Y. M. Gupta, Unloading and reloading response of shocked aluminum single crystals: time-dependent anisotropic material description, J. Appl. Phys. 112 (2012) 093509.

[32] D. Fan, L. Lu, B. Li, M. L. Qi, J. C. E, F. Zhao, T. Sun, K. Fezzaa, W. Chen, S. N. Luo, Transient X-ray diffraction with simultaneous imaging under high strain-rate loading, Rev. Sci. Instrum. 85 (2014) 113902.

[33] L. Lu, D. Fan, B. X. Bie, X. X. Ran, M. L. Qi, N. Parab, J. Z. Sun, H. J. Liao, M. C. Hudspeth, B. Claus, K. Fezzaa, T. Sun, W. Chen, X. L. Gong, S. N. Luo, Note: Dynamic strain field mapping with synchrotron X-ray digital image correlation, Rev. Sci. Instrum. 85 (2014) 076101

[34] M. Hudspeth, T. Sun, N. Parab, Z. Guo, K. Fezzaa, S. Luo, W. Chen, Simultaneous X-ray diffraction and phase-contrast imaging for investigating material deformation mechanisms during high-rate loading, J. Synchrotron Rad. 22 (2015) 49-58.

[35] J. Y. Huang, L. Lu, D. Fan, T. Sun, K. Fezzaa, S. L. Xu, M. H. Zhu, S. N. Luo, Heterogeneity in deformation of granular ceramics under dynamic loading, Scripta Mater. 111 (2016) 114-118.

[36] T. Li, D. Fan, L. Lu, J. Y. Huang, F. Zhao, M. L. Qi, T. Sun, K. Fezzaa, X. H. Xiao, X. M. Zhou, T. Suo, W. Chen, Y. L. Li, M. H. Zhu, S. N. Luo, Dynamic fracture of $\mathrm{C} / \mathrm{SiC}$ composites under high strain-rate loading: microstructures and mechanisms, Carbon 91 (2015) 468-478.

[37] N. D. Parab, B. Claus, M. C. Hudspeth, J. T. Black, A. Mondal, J. Sun, K. Fezzaa, X. Xiao, S. N. Luo, W. Chen, Experimental assessment of fracture of individual sand particles at different loading rates, Int. J. Impact Eng. 68 (2014) 8-14.

[38] D. E. Grady, M. E. Kipp, Continuum modelling of explosive fracture in oil shale, Int. J. Rock Mech. Min. Sci. 17 (1980) 147-157.

[39] R. Pérez, P. Gumbsch, An ab initio study of the cleavage anisotropy in silicon, Acta Mater. 48 (2000) 4517-4530.

[40] D. J. Frew, M. J. Forrestal, W. Chen, Pulse shaping techniques for testing brittle materials with a split hopkinson pressure bar, Exp. Mech. 42 (2002) 93-106.

[41] J. Huang, S. Xu, S. Hu, Influence of particle breakage on the dynamic compression responses of brittle granular materials, Mech. Mater. 68 (2014) 15-28

[42] L. M. Yang, V. P. W. Shim, An analysis of stress uniformity in split hopkinson bar test specimens, Int. J. Impact Eng. 31 (2005) 129-150.

[43] G. McDowell, A. Amon, The application of Weibull statistics to the fracture of soil particles, Soils Found. 40 (2000) 133-141.

[44] Ö. Keleş, R. E. García, K. J. Bowman, Deviations from Weibull statistics in brittle porous materials, Acta Mater. 61 (2013) 7207-7215.

[45] T. Yi, L. Li, C.-J. Kim, Microscale material testing of single crystalline silicon: process effects on surface morphology and tensile strength, Sensor. Actuat. A-Phys. 83 (2000) 172-178.

[46] J. R. Helliwell, J. Habash, D. W. J. Cruickshank, M. M. Harding, T. J. Greenhough, J. W. Campbell, I. J. Clifton, M. Elder, P. A. Machin, M. Z. Papiz, S. Zurek, The recording and analysis of synchrotron X-radiation laue diffraction photographs, J. Appl. Crystallogr. 22 (1989) 483-497.

[47] P. A. Rigg, Y. M. Gupta, Real-time x-ray diffraction to examine elasticplastic deformation in shocked lithium fluoride crystals, Appl. Phys. Lett. 73 (1998) 1655-1657.

[48] T. Sun, K. Fezzaa, HiSPoD: a program for high-speed polychromatic Xray diffraction experiments and data analysis on polycrystalline samples, J. Synchrotron Rad. 23 (2016) doi:10.1107/S1600577516005804

[49] J. Scheibert, C. Guerra, F. Célarié, D. Dalmas, D. Bonamy, Brittlequasibrittle transition in dynamic fracture: an energetic signature, Phys. Rev. Lett. 104 (2010) 045501.

[50] V. Tiwari, M. A. Sutton, S. R. Mcneill, Assessment of high speed imaging systems for 2D and 3D deformation measurements: methodology development and validation, Exp. Mech. 47 (2007) 561-579.

[51] B. Paliwal, K. T. Ramesh, An interacting micro-crack damage model for failure of brittle materials under compression, J. Mech. Phys. Solids 56 (2008) 896-923

[52] H. Inaoka, E. Toyosawa, H. Takayasu, Aspect ratio dependence of impact fragmentation, Phys. Rev. Lett. 78 (1997) 3455-3458.

[53] J. Y. Huang, Y. Li, Q. C. Liu, X. M. Zhou, L. W. Liu, C. L. Liu, M. H Zhu, S. N. Luo, Origin of compression-induced failure in brittle solids under shock loading, Phys. Rev. B 92 (2015) 144101 
[54] D. E. Grady, Fragment size distributions from the dynamic fragmentation of brittle solids, Int. J. Impact Eng. 35 (2008) 1557-1562.

[55] F. Kun, F. K. Wittel, H. J. Herrmann, B. H. Kröplin, K. J. Måløy, Scaling behavior of fragment shapes, Phys. Rev. Lett. 96 (2006) 025504.

[56] C. E. Renshaw, E. M. Schulson, Universal behaviour in compressive failure of brittle materials, Nature 412 (2001) 897-900.

[57] Q. M. Li, H. Meng, About the dynamic strength enhancement of concretelike materials in a split Hopkinson pressure bar test, Int. J. Solids Struct. 40 (2003) 343-360.

[58] M. Zhang, H. J. Wu, Q. M. Li, F. L. Huang, Further investigation on the dynamic compressive strength enhancement of concrete-like materials based on split Hopkinson pressure bar tests. Part I: experiments, Int. J. Impact Eng. 36 (2009) 1327-1334.

[59] W. A. Brantley, Calculated elastic constants for stress problems associated with semiconductor devices, J. Appl. Phys. 44 (1973) 534-535.

[60] A. George, G. Michot, Dislocation loops at crack tips: nucleation and growth-an experimental study in silicon, Mater. Sci. Eng. A 164 (1993) 118-134.

[61] R. Pérez, P. Gumbsch, Directional anisotropy in the cleavage fracture of silicon, Phys. Rev. Lett. 84 (2000) 5347-5350.

[62] G. Hu, K. T. Ramesh, B. Cao, J. W. McCauley, The compressive failure of aluminum nitride considered as a model advanced ceramic, J. Mech. Phys. Solids 59 (2011) 1076-1093.

[63] D. E. Grady, Local inertial effects in dynamic fragmentation, J. Appl. Phys. 53 (1982) 322-325.

[64] M. J. Buehler, H. Gao, Dynamical fracture instabilities due to local hyperelasticity at crack tips, Nature 439 (2006) 307-310.

[65] J. D. Hogan, J. G. Spray, R. J. Rogers, S. Boonsue, G. Vincent, M. Schneider, Micro-scale energy dissipation mechanisms during dynamic fracture in natural polyphase ceramic blocks, Int. J. Impact Eng. 38 (2011) 931939.

[66] R. Weichert, K. Schönert, Heat generation at the tip of a moving crack, J. Mech. Phys. Solids 26 (1978) 151-161. 


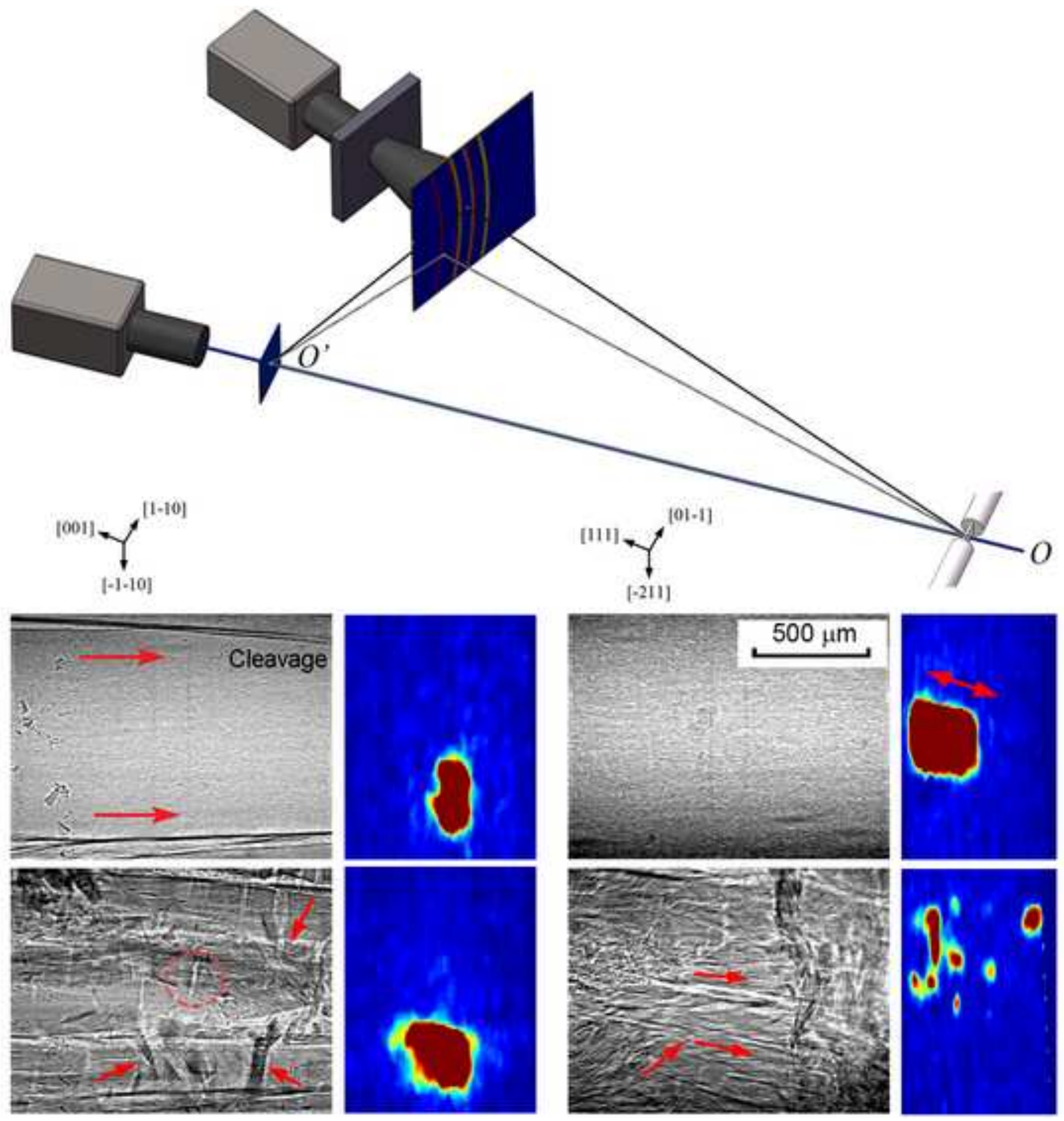

